\title{
¿Qué sucederá con la internacionalización de la educación superior y la movilidad estudiantil después del COVID-19?
}

\author{
Pamela Gómez López \\ Universidad Autónoma Metropolitana - Azcapotzalco \\ Ciudad de México, México \\ https://orcid.org/0000-0002-2346-8074
}

Recibido: 21/09/20 Revisado:01/10/20 Aceptado: 04/11/20 Publicado:05/12/20

\begin{abstract}
Resumen
La internacionalización es un proceso que se lleva a cabo en las instituciones de educación superior con diferentes objetivos, y con su implementación se busca integrar una mirada internacional e intercultural en las funciones de las universidades. Este proceso se compone de diversas estrategias, y, entre las más conocidas, se encuentra la movilidad estudiantil. En las últimas décadas la internacionalización ha cobrado más relevancia, sin embargo, es un proceso que aún carece de un entendimiento integral y se realiza de acuerdo con las intenciones y recursos de las instituciones. Con la llegada de la enfermedad COVID-19, la internacionalización y la movilidad estudiantil se encuentran ante un futuro incierto, ante la urgencia de resolver otras necesidades en las instituciones, por lo que se comienzan a explorar otras formas de implementarla.
\end{abstract}

Palabras clave: Internacionalización de la educación superior; movilidad estudiantil, enseñanza superior; América Latina.

\section{What will happen to the internationalization of higher education and student mobility after COVID-19?}

\begin{abstract}
Internationalization is a process that is carried out in higher education institutions with different objectives and with its implementation it seeks to integrate an international and intercultural perspective in the functions of universities; This process is made up of various strategies, and among the best known is student mobility. In recent decades, internationalization has become more relevant, however, it is a process that still lacks a comprehensive understanding and is carried out in accordance with the intentions and resources of the institutions. With the arrival of the COVID-19 disease, internationalization and student mobility are facing an uncertain future, faced with the urgency of solving other needs in institutions, which is why other ways of implementing it are being explored.
\end{abstract}

Keywords: Internationalization of higher education; student mobility; tertiary education; Latin America.

\section{Qual é o futuro para a internacionalização do educaçao superior e movilizaçao de estudantes depois a COVID-19?}

\section{Resumo}

A internacionalização é um processo que se realiza em instituições de ensino superior com diferentes objetivos e com a sua concretização procura integrar uma perspetiva internacional e intercultural nas funções das universidades; Esse processo é feito de várias estratégias, sendo que entre as mais conhecidas está a mobilidade estudantil. Nas últimas 
décadas, a internacionalização tem se tornado mais relevante, porém, é um processo que ainda carece de uma compreensão abrangente e é realizado de acordo com as intenções e recursos das instituições. Com a chegada da doença COVID-19, a internacionalização e a mobilidade estudantil enfrentam um futuro incerto, face à urgência de resolver outras necessidades nas instituições, razão pela qual estão a ser exploradas outras formas de o implementar.

Palavras-chave: Internacionalização do ensino superior; mobilidade de estudante; ensino superior; América Latina.

Citar como:

Gómez, A. (2020). ¿Qué sucederá con la internacionalización de la educación superior y la movilidad estudiantil después del COVID-19?. Revista Digital de Investigación en Docencia Universitaria. 14(2), e1348. https://doi.org/ 10.19083/ridu.2020.1348

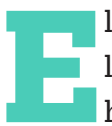
1 proceso de internacionalización dentro de las instituciones de educación superior se ha desarrollado de manera contundente en las últimas décadas. El texto comienza con el planteamiento de su definición conceptual, situando esta práctica como una oportunidad de brindar a los estudiantes y a las instituciones la posibilidad de adquirir competencias que les permitan integrarse dentro de las dinámicas de globalización. Posteriormente, se abordan algunos antecedentes sobre las acciones de las universidades en contexto de pandemia, tomando como precedente el brote de la influenza H1N1 en 2009. Se finaliza con algunas consideraciones sobre los efectos de una nueva pandemia, sobre la internacionalización de la educación superior y la movilidad estudiantil, y algunas opciones que se han colocado en la mesa para dar continuidad al proceso en una era posCOVID-19.

\section{Antecedentes \\ Una aproximación conceptual}

La internacionalización de la educación superior, de acuerdo con una de sus definiciones más conocidas, se refiere a un proceso en donde la dimensión internacional, intercultural y global, o al menos una de ellas, se manifiestan en las funciones de las instituciones de educación terciaria (Knight, 2003). Otra propuesta, enunciada por Altbach, Reisberg y Rumbley (2009), señala que la internacionalización es una respuesta formulada desde los niveles gubernamentales e institucionales para participar dentro del fenómeno de la globalización, lo cual, puede interpretarse como una manifestación del carácter benéfico de la dimensión internacional. Agregando a lo anterior, De Wit (2011), señala que la evolución de la internacionalización ha mutado de una tradición de cooperación a la toma de acciones de competencia entre instituciones de educación superior (IES).

La inclusión de la dimensión internacional en las IES se dirige al alcance de diversos objetivos, que varían de acuerdo con las orientaciones que define cada institución. Sin embargo, como sugiere el análisis realizado en 2012 por Hénard, Diamond y Roseveare para la Organización para la Cooperación y el Desarrollo Económicos (OCDE), uno de los propósitos principales de la internacionalización es la formación académica de calidad para la adquisición de competencias en los estudiantes que les permita interactuar de manera eficaz en el contexto de globalización; el segundo, se refiere al posicionamiento de las instituciones universitarias dentro del mercado educativo mediante la construcción de un perfil internacional que pueda atraer recursos y prestigio, entre otros beneficios.

Para implementar la internacionalización es necesario poner en marcha diversas estrategias que permitan la integración de lo internacional en el nivel de enseñanza superior. Si bien desde las políticas educativas se trazan acciones que permitan la internacionalización, son las instituciones las que se encargan de delinear diversos ejes estratégicos desde los planes de desarrollo institucional para lograr el objetivo principal de este proceso. Sin embargo, esto se encuentra condicionado a las intenciones y recursos financieros con los que se cuenta para llevarlas a cabo. 
Las formas de internacionalizar las instituciones La internacionalización de la educación superior, como es esperado en cualquier proceso, ha evolucionado tanto en su definición como en las estrategias que pueden coadyuvar a su implementación. Para llevarla a cabo, no solo importa el establecimiento de actividades, pues también se debe tomar en cuenta aspectos como el contexto educativo del país y el tipo de institución (De Wit, 2011).

Además, para una internacionalización adecuada, es preciso distinguir sus facetas. En este sentido, son variadas las investigaciones que han realizado énfasis en la diversificación de este proceso. Por una parte, De Wit (2011), señala que la internacionalización puede tomar dos rumbos: uno de ellos, la internacionalización en casa, refiere a las actividades que se llevan a cabo para lograr el desarrollo de la competencia intercultural dentro de los centros universitarios; y la segunda, la internacionalización en el extranjero, considera las acciones que implican la circulación de conocimientos, recursos humanos y establecimiento de diversas redes y convenios, “incluidas las formas de enseñanza más allá de las fronteras" (p. 81).

Alrededor de estos ejes de internacionalización, se pueden encontrar dos vertientes: la internacionalización de carácter endógeno, que alude a la cooperación y reciprocidad entre instituciones; y la internacionalización exógena, permeada por la competencia y los elementos del mercado de la educación, como analizan Oregioni, Taborga y Piñero (2014). Otra propuesta más detallada, la de Knight (s.f.) establece que la internacionalización se realiza considerando cuatro enfoques: actividad, competencia, ethos y proceso, y cada uno de ellos responde a diversas lógicas, entre las que se encuentran la identificación y profesionalización de los actores involucrados en la internacionalización, el establecimiento de una cultura internacional al interior de los campus, la modificación del currículo de manera que incluya la dimensión internacional y, finalmente, el enfoque que vincula cada uno de los ya mencionados para lograr una internacionalización integral.

Uno de los trabajos más relevantes para conocer las estrategias asociadas a la internacionalización es el de Gacel-Ávila. La investigadora distingue diferencias entre las estrategias orga- nizacionales, es decir, aquellas que se plantean desde el nivel institucional y se plasman dentro de los planes de desarrollo institucionales, y las estrategias programáticas, en donde los objetivos de las IES se trasladan a actividades operativas, es decir, aquellas que implican "internacionalizar la docencia, el currículo, la investigación y la extensión" (Gacel-Ávila, 2017, p. 68). Se puede comentar que las estrategias programáticas son la culminación de las intenciones, objetivos y propósitos de internacionalización que tienen las instituciones y centros universitarios.

\section{La movilidad estudiantil, recurso más conocido de la internacionalización}

La movilidad de los estudiantes se ha realizado desde la fundación de las universidades, con propósitos que variaron a lo largo del desarrollo de estas. Desde la Edad Media, los desplazamientos de estudiantes se llevaban a cabo para que accedieran a conocimientos que no estaban disponibles en la universidad de origen. En el siglo XVI, la circulación de estudiantes entró en la categoría de Grand Tour, es decir, viajes que implicaban la construcción o fortalecimiento de relaciones diplomáticas, como indica Ascari (2016). En ambos casos, la movilidad quedaba reservada a la élite y a aquellos que podían costear los gastos de un traslado a otro lugar.

Quizá la estrategia más reconocida es la movilidad estudiantil, que se incorpora al campo de la internacionalización del currículo. Esta se ha definido desde diversos organismos y en innumerables trabajos y, como señala el Instituto Internacional de la Organización de las Naciones Unidas para la Educación, la Ciencia y la Cultura (UNESCO, por sus siglas en inglés) para la Educación Superior en América Latina y el Caribe (IESALC, 2019), la multiplicidad de acercamientos a las actividades de movilidad puede ser acotada a los estudiantes extranjeros que se desplazan a otro país para realizar diversas actividades relacionadas con la ciencia y la investigación.

A medida del surgimiento de nuevos modelos universitarios y la propia evolución de la educación superior, la movilidad estudiantil se realizó con otras directrices. Teichler (2008) menciona que, en el caso europeo, se facilitó en la década de los noventa con la instauración del programa 
Erasmus ${ }^{1}$ y la cooperación entre instituciones universitarias para facilitar no solo la circulación de estudiantes, si no la homologación de créditos y diplomas, y si bien la movilidad se planteó como una opción accesible para la mayoría de los estudiantes, continuó siendo realizada por aquellos con el capital económico suficiente para asumir los gastos del desplazamiento y estadía.

En el contexto latinoamericano, Didou (2017) hace énfasis en que, a pesar de que la movilidad es la estrategia más conocida para internacionalizar la educación, a nivel regional aún está en un nivel muy por debajo de los números que arrojan las encuestas realizadas en otros lugares. Por otra parte, un indicador que salta a la luz, de acuerdo con $\mathrm{Di}$ dou, es que los estudiantes extranjeros matriculados en instituciones latinoamericanas no suman un porcentaje alto. Un aporte fundamental de la investigación realizada por la autora, resalta otras problemáticas inmersas en la decisión de movilidad, como el aprendizaje de un idioma, la inmersión intercultural para una adaptación exitosa en la universidad receptora y las becas, en ocasiones insuficientes, para solventar los gastos de los estudiantes mientras se encuentran fuera del lugar de origen.

La definición de movilidad también ha sido objeto de diversas modificaciones. Una interpretación es ofrecida por Donelly y Gamsu (2018) en donde explican que una definición de movilidad puede ser construida desde la medición de distancia entre el lugar de origen y la universidad destino. El aporte de Góngora (2018), en tanto, hace énfasis en la temporalidad de las movilidades, mientras que Jung (2016) señala que, en el tema de la movilidad, existen diversos sentidos "algunos empíricos, otros ideológicos, así como fenómenos que sobre los que los estados occidentales son susceptibles de incidir y otros pertenecen al

1 Erasmus es el programa de intercambio estudiantil, activo desde la década de los ochenta, para los países miembros de la Unión Europea. La transferencia y homologación de créditos, así como el componente intercultural e internacional son dos de los atractivos que ofrece este programa; ofrece además financiamiento para la estancia de los estudiantes y la diversificación de los propósitos del programa, como voluntariados, trabajos, estudio de asignaturas y programas de entrenamiento en diversas instituciones. Disponible en https:// www.erasmusprogramme.com/ registro de lo simbólico" (p. 21).

En suma, la movilidad estudiantil implica diversos factores y su definición estará ligada a los propósitos que para ella se tengan. La propuesta que realizó radica en establecer que la movilidad estudiantil, nacional e internacional, es un desplazamiento desde la universidad de origen hacia una universidad destino, con el propósito de realizar una estancia con diferentes temporalidades y diversos propósitos, como el curso de asignaturas, trabajo de campo, inserción en un proyecto o grupo de investigación, entre otras. Se considera además que la movilidad, si bien se realiza desde la lógica individual, se enmarca en las disposiciones institucionales que, en gran medida, posibilitan o no el tránsito de los estudiantes.

La definición de la UNESCO ${ }^{2}$ describe que los estudiantes se vuelven internacionalmente móviles cuando cruzan fronteras para residir de manera temporal en un país diferente al que se encuentran estudiando, con el objetivo de realizar actividades académicas. De acuerdo con la definición anterior y los datos de International Organization for Migration's Global Migration Data Analysis Centre (GMDAC) el número de estudiantes móviles en el periodo 2011-2017 fue de casi cinco millones. La mayor movilidad saliente se reporta en Norteamérica, Europa y Asia, mientras que Latinoamérica, el Caribe y Oceanía tienen un menor número de estudiantes de movilidad, como reflejan los reportes de la UNESCO Institute for Statistics ${ }^{3}$ Por otra parte, respecto a los destinos elegidos por los estudiantes, se mantiene la misma constante que en la movilidad saliente, ya que Norteamérica, países europeos y asiáticos reciben más estudiantes internacionales por año.

Es así como podemos observar que la internacionalización y su estrategia más conocida e implementada, la movilidad estudiantil, han sido procesos presentes desde la fundación de la universidad. Dentro del reporte Trends in Global Higher Education: Tracking an Academic Revolution para la Conferencia Mundial en Educación Superior organizada por la UNESCO, Altbach,

2 http://uis.unesco.org/en/glossary-term/international-or-internationally-mobile-students

3 https://migrationdataportal.org/infographic/internationally-mobile-students 
Reisberg y Rumbley (2009) señalaban que la vorágine de la globalización había propiciado que las universidades fueran más competitivas, en la búsqueda de mantenerse dentro de los rankings ${ }^{4}$ que, en cierta forma, constriñen el imaginario de los actores educativos y por tanto, su inclinación a elegir o no determinada universidad para la continuación o complemento de los estudios. Por supuesto, la internacionalización podría ser un factor atractivo para la imagen de las instituciones.

Lo anterior, busca brindar un panorama general del proceso de internacionalización de la educación superior y la movilidad estudiantil. La educación superior, a lo largo del tiempo, ha modificado varios de sus procesos y, en su masificación, la matrícula ha llegado hasta los 250 millones de estudiantes inscritos en los diversos programas de educación terciaria y de estos, la tasa de movilidad es de aproximadamente $2 \%$ (considerando el dato presentado anteriormente, de los casi 5 millones de estudiantes móviles, Calderon, 2018).

Sin embargo, a pesar del enfoque atractivo que tiene la internacionalización, en años recientes se ha advertido que este proceso se encuentra en un punto de riesgo. Las estrategias compartidas como la construcción de redes de investigación internacionales, cátedras, eventos culturales y movilidad académica, entre otras, encontraron un relativo equilibrio sin mayor crecimiento dentro de las instituciones universitarias, al agotarse, en medida, la diversificación de estrategias al concentrarse en el impulso de solo algunas de ellas y mantener la visión concentrada en un solo tipo de internacionalización.

Además de ello, las cuestiones políticas han jugado un papel determinante en la desaceleración de algunas acciones para el establecimiento de la dimensión internacional. Por una parte, el crecimiento de movimientos políticos y sociales, aunado a actitudes ostracistas hacia los individuos de origen extranjero que han ido en aumento en los últimos años, han puesto en entredicho la facilidad

4 Downing, Lock y Leung (2018) indican que la historia de los rankings universitarios puede rastrearse hasta la década de los ochenta en Estados Unidos. Un ranking tiene diferentes indicadores que, en conjunto, buscan demostrar la calidad de las instituciones, basados en el número de matrícula, programas educativos, eficiencia terminal e incluso, internacionalización. para realizar trámites migratorios, especialmente para aquellos que no radicarán por un periodo largo en un país de acogida, como el caso de los estudiantes móviles. Enseguida, como señalan Altbach y De Wit (2018), se suman las condiciones que algunos países han implementado para filtrar de manera más estricta el ingreso de estudiantes a las instituciones universitarias, las acciones para reducir el uso del inglés como lenguaje científico y los costos de matrícula para estudiantes extranjeros.

Algunos otros especialistas, señalan también que otra de las problemáticas que encuentra la internacionalización es el no retorno al país de origen (Remedi \& Ramírez, 2016), las crisis económicas y el decrecimiento de los recursos otorgados para la cooperación internacional (Jaramillo, 2016) e incluso el "colonialismo científico que podría estar implícito en las políticas de cooperación" (Schwartzman, 2009, p. 69).

A pesar de estas circunstancias, que podrían tener un peso negativo para la internacionalización de la educación superior, autores como Didou (2013) mencionan que en el tema de movilidad, si bien hay vacíos en los datos sobre número de estudiantes y académicos móviles, condiciones de los estudiantes extranjeros y la validación de créditos al término del desplazamiento, es posible apelar al lado positivo del proceso, como la continua colaboración entre especialistas de diversos campos disciplinares para la difusión de conocimientos. En tanto, Camacho (2017) menciona que las razones fundamentales para la internacionalización pueden devenir en un impacto positivo para el establecimiento de convenios y alianzas, y la formación integral, internacional y personal de los estudiantes que realizan movilidad.

\section{Discusión}

\section{La educación superior en tiempos de pandemia}

El 11 de marzo de 2020, se realizó la declaratoria de pandemia por parte de la Organización Mundial de la Salud (OMS). Otras pandemias, como la provocada por el Síndrome Respiratorio Agudo Grave (SARS) y la Influenza H1N1, habían propiciado la emisión de comunicados para alertar acerca de la 
emergencia y las medidas para frenar la expansión de la enfermedad. Al mes de marzo, se habían reportado 118000 casos y 4291 muertes. Para el mes de agosto, la cifra aumentó a 23, 752, 965 casos y 815,038 decesos ${ }^{5}$.

En la época reciente, fue la última enfermedad la que representa el precedente más cercano que detonó acciones en distintos ámbitos que se llevaron a cabo para superar la emergencia. El brote de influenza AH1N1 fue detectado en México en 2009. La emisión de distintas notas informativas por parte de la OMS durante el desarrollo de esta enfermedad destacaba las medidas sanitarias, el listado de síntomas, el riesgo de propagación y las recomendaciones para la población que había contagiado, entre otros tópicos (Reynoso, 2010). $\mathrm{El}$ autor mencionado indica que dentro de estas notas existía una referencia especial para la suspensión de actividades escolares, ya que estos espacios representaban zonas de alto riesgo de contagio por el número de personas dentro de los recintos educativos, por lo cual se acató esta medida en distintos países, especialmente México, por ser el epicentro del origen de la influenza.

Para el 11 de septiembre de 2009, la OMS realizó la difusión de otra nota informativa, indicada para los establecimientos escolares, en donde se manifestaba el reforzamiento de las medidas preventivas sanitarias $\mathrm{y}$, además, recomendaba el cierre de escuelas para la detención significativa del aumento de casos, aunque tal recomendación se dejó en manos de los diferentes gobiernos.

En el caso de la educación superior, particularmente en la movilidad estudiantil, las medidas establecidas fueron el retorno de estudiantes y la suspensión temporal de programas de intercambio y estancias fuera del país de origen. Además, ante la detección de casos entre los estudiantes, la medida más conveniente fue mantenerse en cuarentena y como indica Redden (2009) en algunas instituciones norteamericanas si bien no existió cancelación de los viajes estudiantiles, ante la decisión de los estudiantes de viajar a México se ofrecían dos posibilidades: una, realizar la estancia manteniendo un contacto continuo con tuto-

5 De acuerdo con las cifras presentadas en WHO Coronavirus Disease (COVID 19) Dashboard. Disponible en https:// covid19.who.int/ res y universidades; y la otra, quizá la más pertinente, elegir otro país receptor.

Otra mirada de las medidas preventivas aplicadas en escuelas proviene del estudio de caso de Katz, May, Sanza, Johnston y Petinaux (2012) en donde, a partir del análisis de la actuación de autoridades escolares ante la evolución de la influenza H1N1, se recurrió, en un primer momento, a la difusión de medidas de prevención mediante el uso de tecnologías de la información y la comunicación, la vacunación preventiva y la implementación del uso del cubrebocas. Aunque esta última acción no fue del particular agrado de los estudiantes en tanto que el distanciamiento social fue una de las acciones más aceptadas.

Lo anterior, conduce al reporte H1N1 and Higher Education. Lessons learned: tools, tips, takeaways elaborado por el Center for Infectious Disease Research (CIDRAP, 2010) de la Universidad de Minnesotta, en donde se informó que, en los primeros casos detectados de estudiantes infectados por H1N1, se conoció que probablemente contrajeron el virus en los viajes denominados spring break. Pero una particularidad para la respuesta de ciertas instituciones norteamericanas fue la preparación para el escenario de pandemia, tomando como base las diferentes enfermedades surgidas en Asia que tenían el potencial de convertirse en padecimientos letales.

Así, dicho reporte se pudo incluso interpretar como un manual para la implementación de diversas medidas de contención para una situación emergente. En el caso de la movilidad estudiantil o study abroad fue cancelada para México, pero no hacia otros países y los estudiantes móviles extranjeros podían recurrir al denominado emergency housing ${ }^{6}$ ante la imposibilidad de retornar a sus países.

En la revisión de la literatura asociada a las pautas de actuación de las universidades durante esta pandemia, no fue posible corroborar las acciones llevadas a cabo por continente, país o institución.

6 Dentro del reporte mencionado, emergency housing refiere a la construcción de soportes institucionales y comunitarios para el hospedaje de estudiantes extranjeros en el país receptor. Si bien se indica que dicho plan no requirió su activación dentro del desarrollo de la pandemia por influenza, funcionó para fortalecer la cohesión entre universidad-sociedad. 
Pese a ello, no se encontró información de la afectación de la enfermedad a los planes de internacionalización, pero en el caso de la movilidad estudiantil, se infiere que la expansión mundial de dicha enfermedad tuvo un efecto similar en las acciones de desplazamiento en un alto porcentaje de universidades, ya que la prioridad era mantenerse alejado del territorio de origen de la influenza, modificar la elección de universidad receptora o suspender de manera temporal cualquier actividad académica fuera del país de origen.

Por otra parte, el cierre preventivo de los centros escolares, incluidas las universidades, obligó a crear protocolos de acción que proporcionaran en el corto plazo una solución ante el panorama incierto y la evolución de la pandemia. Sin duda, proporcionar información sobre la enfermedad fue una de las primeras acciones que se llevaron a cabo. A partir de este conocimiento, se podrían delinear estrategias que permitieran abordar la problemática de los cursos suspendidos. Una de las soluciones inmediatas fue la adopción de los denominados MOOC (Massive Online Open Courses) que, como señala Trucano (2014), se consideraban una opción viable, de bajo costo y de acceso relativamente sencillo para la continuación de estudios, aunque en el trasfondo, el desplazamiento a este tipo de educación requería planeación y capacitación docente para un uso efectivo de las tecnologías de la información y la comunicación (TIC), y el desarrollo de programas educativos que pudieran ser facilitados de manera virtual.

Para la movilidad estudiantil, la acción más drástica fue cancelar, suspender o reprogramar las estancias de los estudiantes en otros países, especialmente en México. De manera sucinta, se puede comentar que la contingencia sanitaria no representó un cambio impactante dentro de la internacionalización de la educación superior. Sin embargo, funcionó como un mecanismo de atención a los protocolos implementados por las instituciones de diferentes niveles ante casos de emergencia.

\section{La irrupción del COVID-19}

La propagación de la enfermedad COVID-19 a principios de este año, nuevamente puso la alerta sobre las diversas medidas para mitigar sus efectos. Así, los comunicados de distintas organizaciones se centraron no solo en difundir la gravedad del
COVID-19, si no las diversas estrategias para controlarla. Por supuesto, las consecuencias sociales, económicas y políticas surgieron de forma inmediata. Las medidas para mitigar el brote de la enfermedad, ante el desconocimiento de la forma de propagación de esta, incluyeron el lavado de manos, el distanciamiento social y las diversas indicaciones para evitar los lugares concurridos. Posteriormente, comenzó un cierre paulatino de negocios, centros recreativos, lugares de esparcimiento y escuelas de todos los niveles, incluyendo las instituciones de educación superior.

La limitación de actividades en las universidades, incluidas las labores administrativas, fue una medida de prevención que, hasta cierto punto, atendía la indicación proveniente de los organismos de salud, nacionales e internacionales, de mantener un estricto distanciamiento social. Sin embargo, otra problemática, sumada a la suspensión de ciclos escolares e intercambio a las actividades en forma virtual, estaba por alertar a las instituciones de educación superior: las estancias de académicos y estudiantes en el extranjero.

A lo largo del mundo, las embajadas comenzaron a emitir alertas y restricciones de viaje por el coronavirus, además del cierre de fronteras para evitar al máximo el número de contagios. Estas acciones se llevaron a cabo de manera escalonada, pues no en todos los países existían casos o directrices claras que atender. A través de la realización de vuelos humanitarios, se llevaría a cabo la repatriación de personas a sus lugares de origen o vivienda. A diferencia de la situación de 2009, en esta ocasión se hizo énfasis en la urgencia de limitar la circulación de personas y planificar la vuelta a casa de aquellas personas que se encontraban fuera de su lugar de origen, a través de vuelos humanitarios. Otra de las posibilidades fue la permanencia en el país extranjero durante el tiempo de contingencia.

\section{Notas sobre el desarrollo de la internacionaliza- ción en la educación superior latinoamericana}

Al respecto del rubro de internacionalización, Gacel y Rodríguez (2018) indican que las razones para internacionalizar difieren por país, por institución y se condicionan por las estructuras de gestión institucional. En coincidencia con Marmolejo (2018), mencionan que en la actualidad el presu- 
puesto para internacionalización ha aumentado y, aunque se diversifican actividades para la atracción de recursos, las instituciones públicas de educación superior aun dependen de los recursos públicos para sostener las actividades de carácter internacional, ya que se enfrentan a algunos riesgos y obstáculos para llevarlas a cabo, entre ellas, el entendimiento poco claro de las estrategias propuestas para lograr incluir la visión internacional en las instituciones.

Un panorama más claro de la internacionalización en la región se encuentra plasmado en la "I Encuesta Regional sobre tendencias de la internacionalización en América Latina y el Caribe" realizada en el 2016 por el Observatorio Regional sobre Internacionalización y Redes en Educación Terciaria en América Latina y el Caribe (OBIRET, 2018). De manera particular, habrá que remitirse a los obstáculos para la internacionalización para comprender posteriormente, como se ha visto afectada por la emergencia sanitaria. Así, Gacel-Ávila y Rodríguez-Rodríguez (2018) mencionan dos tipos de obstáculos para la inclusión de la práctica internacional en las actividades, detallando:

\section{- Obstáculos internos (o institucionales)}

Son aquellos que se encuentran inmersos estructuralmente en las instituciones y se vinculan con cuestiones económicas, como el financiamiento; académicas, relacionadas con la falta de integración en el currículo y el aprendizaje de idiomas; y administrativas, que refieren a la falta de capacitación en temas relacionados con este proceso, por parte de los encargados de las oficinas de internacionalización.

\section{- Obstáculos externos}

Financiamiento público insuficientes, falta de claridad en los procesos de homologación y validación de créditos, y restricciones de visa (para movilidad saliente y entrante)

El análisis de Gacel-Ávila y Rodríguez-Rodríguez (2018) proporciona las pautas que ayudan a comprender las afectaciones de la pandemia en el ámbito institucional para la internacionalización de las instituciones terciarias. Algunos de los informes que han cobrado gran relevancia son los emitidos por la UNESCO, en conjunto con la Comi- sión Económica para América Latina y el Caribe (CEPAL, 2020), en donde ha quedado plasmado que la suspensión de clases es, en casos de emergencia, la medida más adecuada para disminuir afectaciones.

Sin embargo, es necesario reconocer que la acción anterior se llevó a cabo a nivel mundial y regional, por tanto, no fue una estrategia específica para las instituciones de educación superior. La internacionalización pasó a un segundo plano ante necesidades de mayor urgencia en las universidades, como la continuidad de los ciclos escolares y los procesos de admisión. Algunas sentencias, como las emitidas por Francisco Marmolejo y Renato Balderrama en la segunda edición del foro Retos y oportunidades para la internacionalización de la educación superior, organizado en mayo de 2020, por la Asociación Mexicana para la Educación Internacional (AMPEI), señalaban que, en efecto, la pandemia arrojó luz sobre las complejidades de la equidad en el acceso y la permanencia en este nivel. Sin embargo, el tema de internacionalización fue minimizado ante las restricciones de viaje que, en definitiva, ponían en paro distintas estrategias, entre ellas, la movilidad de académicos y estudiantes.

Lo anterior parecería colocar el papel de la internacionalización muy por debajo de su potencial de apoyo para la cooperación y colaboración entre países y universidades. Pese a ello, este proceso se ha posicionado como un must de la globalización. De acuerdo con la opinión de Marmolejo emitida dentro del foro de la AMPEI, ante una situación como la que se está viviendo, es posible que la internacionalización pueda tener nuevos entendimientos y con ello, derribar las connotaciones negativas que se le atribuyen. Sylvia Ortega, en tanto, dentro del mismo espacio de discusión, refirió que la internacionalización debe ser disruptiva, encaminada a la construcción de proyectos regionales. A manera de conclusión de este apartado, podemos considerar que la internacionalización de las universidades se encuentra en un momento frágil. Indiscutiblemente, sus ventajas y desventajas son diversas, aunque se hace innegable que, gracias a su implementación, se ha logrado el acercamiento entre instituciones que promueven ideales de cooperación. Por otra parte, es determinante que en un escenario post pandemia, de nueva cuenta 
se propicien acciones que coadyuven al entendimiento de la internacionalización en el ámbito educativo y se realicen propuestas que incluyan otras posibilidades de internacionalizar, además de la movilidad.

\section{La suspensión de los desplazamientos académi- cos y estudiantiles}

Hasta ahora, se han brindado algunos acotamientos acerca del futuro de la internacionalización. En ese tenor, es de suma relevancia reconocer las prospectivas de la movilidad estudiantil en el presente y el posible giro que tendrá en el futuro.

La movilidad estudiantil refiere a los desplazamientos temporales de los estudiantes a universidades destino, para llevar a cabo diferentes actividades académicas. De acuerdo con el reporte "La movilidad en la educación superior en América Latina y el Caribe: retos y oportunidades de un convenio renovado para el reconocimiento de estudios, títulos y diplomas" firmado por el IESALC (2019), el porcentaje de estudiantes móviles con destinos internacionales y dentro de la región en el periodo 2012-2017, alcanzó un 2.3; "en cifras: de 4 a 5 millones en solo cinco años" (p. 11).

Si bien la cifra es baja comparándola con los números de la movilidad en otros continentes y regiones, no se ha estancado y aumenta de manera paulatina. Tal como muestra el reporte, las elecciones de movilidad se dirigen a escoger destinos como Estados Unidos y Europa para las experiencias de desplazamiento, aunque también los destinos latinoamericanos, como México, Brasil y República Dominicana, se erigen como atracción para estudiantes extranjeros.

Desde el cese de actividades universitarias, los flujos de estudiantes también entraron en interrupción y diferentes acciones se tomaron para proporcionar una solución para la movilidad entrante y saliente: en algunos países se les brindó alojamiento, apoyo en trámites migratorios y continuidad de actividades en línea. Pero no todo fue positivo: algunos quedaron varados en los destinos elegidos para su movilidad, imposibilitados de realizar trámites para regresar a casa, con precariedad económica y posiblemente, con efectos negativos en su estado anímico.

Algunas reflexiones fueron recuperadas para este apartado. En principio, la de Altbach y De Wit
(2020) manifestaron que habría un descenso en el número de estudiantes móviles ante un periodo de incertidumbre en cuanto a la reanudación de actividades en las universidades. Recuperan las ideas de Marginson para así proyectar la recuperación de los flujos de estudiantes móviles dentro de cinco años, pero con cambios significativos en la elección de destinos y la disminución del financiamiento para realizar estancias al extranjero.

Las consecuencias del COVID-19 no solo se encuentran en la pausa de la movilidad estudiantil. La crisis económica mundial incide negativamente en el presupuesto previsto para la educación como indica Schleicher (2020), tomando en cuenta los reportes de la Organización para la Cooperación y el Desarrollo Económicos (OCDE). Además de ello, dentro de este detrimento económico se incluye la pérdida de ingresos de cuotas de los estudiantes y los beneficios de incrementos en el producto interno bruto, como ha estudiado Pawlowska (2011) en su investigación acerca de la derrama económica de los estudiantes de movilidad como participantes del turismo cultural. De igual manera, Quinteiro (2020) reconoce que el factor económico será trascendental para la continuidad de las acciones de movilidad, en tanto existirán incrementos en el transporte aéreo y el presupuesto institucional destinado a la movilidad.

Las ventajas y desventajas de la movilidad estudiantil han sido definidas en diferentes investigaciones, pero a pesar de algunos riesgos que representa (como la posible fuga de cerebros), se ha considerado como una actividad que puede favorecer la adquisición de las llamadas competencias globales ${ }^{7} \mathrm{y}$, como valor agregado, la competencia intercultural ${ }^{8}$. En el campo académico, la

7 De acuerdo con la definición del Programme for International Student Assesment (PISA) la competencia global es la "capacidad de examinar asuntos locales, globales e interculturales, para entender y apreciar las perspectivas del mundo y las perspectivas de otros, vincularse en interacciones apropiadas, abiertas y efectivas con personas de diferentes culturas y actuar por el bien colectivo y el desarrollo sustentable" (Teaching for Global Competence in a Rapidly Changing World. https://asiasociety.org/ sites/default/files/inline-files/teaching-for-global-competence-in-a-rapidly-changing-world-edu.pdf).

8 Han sido definidas como las habilidades que favorecen una perspectiva de reconocimiento de la diversidad de 
movilidad es considerada como una actividad que puede permitir la construcción de redes de investigación, el acercamiento a nuevas perspectivas teóricas y, en general, puede calificarse como una experiencia positiva. El cese de los desplazamientos, como se ha abordado, tiene impacto en distintos ámbitos y, por tanto, también detiene los encuentros culturales, de gran importancia para los estudiantes móviles, por lo que además de la adecuación de los programas de movilidad, se presenta como una nueva necesidad sustituirlos recurriendo al uso de la tecnología, hasta el momento en que se reanuden los encuentros presenciales.

La tecnología como recurso de internacionalización: traer al otro mediante recursos virtuales A partir del establecimiento de las medidas de distanciamiento social para contener los contagios de COVID-19, nuestras vidas y cotidianidades en gran medida se han visto inmersas en la tecnología sustituyendo la presencia física por una presencia virtual que favorece los procesos de socialización y comunicación.

En las escuelas y centros universitarios, las clases se han sustituido por cursos en línea que buscan dar continuidad a los ciclos escolares, tanto en inicio como en término. La UNESCO estableció diversos mecanismos: la enseñanza en los rubros de recursos, software, cursos en línea, plataformas de comunicación en video, acceso a repositorios y herramientas para docentes ${ }^{9}$, y varios sistemas educativos a nivel mundial optaron por enseñanza remota.

Pero esta transición descubrió una brecha de desigualdad en la que se reconoce que no todos los estudiantes tienen acceso a internet y no cuentan con los dispositivos tecnológicos para continuar con su educación. En el caso de las instituciones, se ha revelado que es insuficiente la capacitación de docentes en el campo de la tecnología y, además, se carece de estrategias adecuadas

culturas y estilos de vida que permiten la interrelación y comunicación efectiva entre los individuos. Retomado de Competencias interculturales. Marco conceptual y operativo. UNESCO - Universidad de Colombia. https:// unesdoc.unesco.org/ark:/48223/pf0000251592

9 https://en.unesco.org/covid19/educationresponse/solutions para transitar a la enseñanza virtual. Añadiendo a lo anterior, Barrón (2020) señala que es necesaria una reforma estructural de la educación en la que la tecnología pueda ser incluida como un recurso pedagógico; baste añadir que solo mediante su uso encaminado a generar buenas prácticas para el proceso de enseñanza-aprendizaje se puede hablar de un aspecto positivo del uso de las TIC en educación y no solo como mera herramienta de transmisión de contenidos que no cobren significancia para los estudiantes.

En el caso de la educación superior, las medidas han sido las mismas que en otros niveles educativos: optar por la enseñanza a distancia, en tiempos de pandemia, ha sido quizá la única alternativa viable para instituciones y actores educativos. La suma de otros retos como el acceso, cobertura y permanencia en el nivel superior sin duda han sido relevantes en este nuevo contexto y probablemente los retos se multipliquen una vez superada la contingencia. De momento, las universidades y demás centros que conforman el nivel educativo superior han tenido que migrar a los espacios virtuales para continuar atendiendo a los millones de estudiantes afectados por la pandemia en la región latinoamericana.

A propósito de lo anterior, Toro (2020) mantiene que hay dos panoramas en la educación terciaria: uno, de instituciones que ya habían transitado por la experiencia escolarizada en línea que pudieron atender de manera rápida las demandas estudiantiles; y el segundo, que señala la urgencia de la incursión virtual de las instituciones que, en definitiva, requieren de esfuerzos institucionales y de los docentes para mantener los espacios de aprendizaje. En el área de la educación internacional, el investigador refuerza la idea de que la internacionalización en casa es la estrategia más conveniente para adquirir competencias globales cuando no es posible trasladarse de manera física a otros contextos educativos. En tanto, Gacel-Ávila (2020) enfatiza que, en efecto, el fortalecimiento de la internacionalización en casa puede dar espacio para la creación de estrategias novedosas y reformulación de los currículos, aunque primero, habría que resolver el problema del poco entendimiento de esta faceta del proceso de internacionalización desde el nivel institucional y administrativo.

Así, la internacionalización en casa que enfati- 
za la adquisición y desarrollo de las competencias globales sin necesidad de desplazamientos (Beleen, 2011), y la inclusión de la dimensión internacional en el currículo, investigación y actividades académicas (Gacel-Ávila, 2017), se comenzó a explorar más a fondo para reconocer sus bondades dentro de las universidades. Pero de nueva cuenta, dentro de muchas instituciones, particularmente las latinoamericanas y de países subdesarrollados, la internacionalización no es un tema de urgencia en las agendas, aunque sin duda, puede ser objeto de discusión una vez que se trascienda la situación actual. Por otra parte, Schleicher (2020) pone sobre la mesa el hecho de que, para los estudiantes que buscaban realizar estancias en el extranjero, no podría resultar atractivo el cambio a la modalidad virtual, puesto que elimina la posibilidad de la experiencia cara a cara con otros contextos.

Respecto a esta respuesta y ante la imposibilidad de trasladarse físicamente a otras universidades para complementar la formación académica, ha resurgido el interés por el denominado COIL (Collaborative Online International Learning). Este tipo de aprendizaje, desde la óptica de la pedagogía, alude a "fomentar el desarrollo de competencias interculturales con el uso de tecnología para conectar aulas en distintas ubicaciones geográficas" (Appiah-Kubi \& Annan, 2020, p. 109) de manera que los estudiantes pueden confrontarse con otras perspectivas culturales y académicas sin necesidad de desplazamientos, lo cual, además, brindaría oportunidad a los estudiantes menos favorecidos de acceder a la experiencia de internacionalización.

Uno de los estudios recientes sobre este tema, "How Covid-19 has affected young universities", firmado por Gomez y Colella (2020), señala que entre las estrategias de COIL se pueden encontrar las mentorías virtuales y entrenamientos interculturales. Ambas pueden sostener las redes de colaboración internacional y fructificar los esfuerzos de internacionalización mediante la enseñanza de idiomas y tutorías mientras que el impulso a la movilidad virtual puede construir un terreno de transición para la experiencia presencial que pueda facilitar la adaptación de los estudiantes a nuevos escenarios.

La investigación de Levinson y Davidson (2015) arrojó resultados positivos respecto a la movili- dad virtual, clasificados en tres categorías: experiencias virtuales con equipos internacionales en búsqueda de la elaboración de proyectos; las experiencias híbridas, es decir, vincular las actividades virtuales con las presenciales; y, finalmente, las actividades extendidas que buscan prolongar la experiencia de movilidad virtual a lo largo de los estudios. Las actividades mencionadas no solo requieren de un plan de internacionalización institucional bien cimentado, pues habrá que añadir que solo pueden funcionar de forma adecuada si existe colaboración entre docentes, de par a par, y se ajustan a los husos horarios, pues si bien se establecen grupos de trabajo internacionales, no todos los estudiantes pueden estar presentes de forma sincrónica en las sesiones virtuales.

De manera más precisa, Guth, Doscher y Haug (2020) señalan que el método COIL (traducido al español como Aprendizaje Colaborativo Internacional Online) no es nuevo, pero al igual que la internacionalización, requiere un entendimiento de la terminología asociada a sus estrategias y, más aún, se debe trascender del término de movilidad virtual hacia el intercambio virtual que finalmente precisa de manera más fina uno de los objetivos de COIL, que es la interacción y diálogo asertivo entre pares que puedan resultar en experiencias que detonen la competencia intercultural.

Una de las alternativas en Latinoamérica es el Espacio de Movilidad Virtual en Educación Superior (eMOVIES) ${ }^{10}$ impulsada por la Organización Universitaria Interamericana que ofrece a los estudiantes la opción de tomar cursos en universidades extranjeras y apostando por la homologación de créditos en la institución de origen, lo cual refleja la intención de algunas instituciones en la región por equilibrar el acceso a las experiencias internacionales, eliminando el pago de matrícula, pero prescinde en todo momento de las experiencias presenciales en concordancia con el carácter virtual del programa; la alternativa de movilidad virtual ha sido adoptada también por el Programa Erasmus, uno de los más reconocidos en el mundo por la experiencia cultural y académica que brinda a los estudiantes.

10 Espacio de Movilidad Virtual en la Educación Superior (eMOVIES). Disponible en: https://oui-iohe.org/es/emovies/ 
Desde luego que integrar innovaciones de internacionalización dentro de los planes de desarrollo institucional (o al menos en los protocolos para emergencias como la que ahora se vive) requiere esfuerzo a nivel institucional, de programas académicos y finalmente, de los propios estudiantes. Por otra parte, es inevitable pensar que la movilidad virtual no es suficiente para el desarrollo de distintas competencias en los estudiantes, aunque hay que reconocer que propicia la inclusión de los estudiantes en un proceso que se ha formulado como una respuesta a las demandas de la globalización.

Reflexiones finales: ¿qué futuro espera para la internacionalización y la movilidad estudiantil? La irrupción del COVID-19 en el mundo ha tenido consecuencias devastadoras. Los sectores científico, económico, político, social y educativo han sufrido el embate de una enfermedad que ha obligado a repensar muchas de nuestras prácticas y adquirir otras para adaptarnos a un mundo donde la normalidad es, quizá momentáneamente, suspender los encuentros cara a cara que nos han obligado a recurrir a la tecnología como una especie de artefacto que nos mantiene cercanos en un escenario donde el distanciamiento social es la nueva norma.

Desde luego que el ámbito escolar ha sido uno de los terrenos más afectados. Las múltiples texturas de los sistemas educativos de cada país han fomentado decisiones drásticas, como el cierre de escuelas y la entrada forzosa al aprendizaje en línea que buscaba ser equitativo e igualitario $\mathrm{y}$, paradójicamente, no se ajusta a las realidades de miles de estudiantes que debido a múltiples factores no pueden continuar con las clases a distancia.

En el tema de internacionalización, diversas voces han manifestado que este proceso ha pasado al último escalón de relevancia, pues como en reiteradas ocasiones se menciona

lo largo de este escrito, la urgencia se inclina a la planificación sobre la marcha de estrategias que permitan continuar con los procesos de aprendizaje de los estudiantes.

La colaboración entre países se ha observado en los últimos meses a través de las diversas acciones que se llevan en conjunto para encontrar la cura al virus que ha cobrado la vida de miles de personas. Esa misma colaboración se ha llevado al terreno de la educación superior, donde las redes de cooperación, convenios, coautorías, integración de equipos internacionales y recepción de estudiantes extranjeros en todas las latitudes del planeta.

La internacionalización, presente en la historia de las universidades, ha sido uno de los procesos que continuamente se diversifican, a la par de un mundo cambiante. El futuro de esta práctica requiere una mayor flexibilidad en su implementación y estrategias que, ante este panorama, se concluye que no solo deben remitirse a la movilidad estudiantil, pues en favor de acciones de igualdad y equidad, pueden direccionarse hacia nuevos escenarios en donde todos puedan participar de encuentros significativos con otras culturas.

Hasta el momento, de acuerdo con los mapas elaborados por The British Council ${ }^{11}$ las instituciones de educación superior en gran parte del mundo mantienen su cierre por lo que la interacción cara a cara queda relegada solamente al terreno de lo virtual. Otros datos proporcionados por el mismo consejo, indican que en este mes (septiembre de 2020) ha incrementado el flujo de estudiantes, lo cual no quiere decir que las restricciones de viaje hayan terminado, si no que refleja la vuelta de estudiantes a sus países de origen y la aceptación de algunos otros en universidades al extranjero, pero no de manera presencial, a la espera del reinicio de actividades presenciales que aún parece lejano.

Algunas recomendaciones establecidas por la UNESCO enfatizan que hoy más que nunca, los lazos de cooperación deben estrecharse entre instituciones universitarias. Por tanto, repetimos que estas acciones pueden afianzarse ante el nuevo embate que el próximo año deberá enfrentar el sector educativo (y otros tantos): la crisis económica derivada de la emergencia sanitaria.

En suma, la educación superior, la internacionalización y la movilidad estudiantil se encuentran en un momento que puede permitir que se renueven sus procesos, a la luz de una nueva normalidad que obliga al acercamiento virtual. Pese a lo negativo de estas circunstancias, habrá que pensar que

11 https://www.britishcouncil.org/education/ihe/news/international-student-mobility-heatmap 
las intenciones de viajar, conocer nuevos ambientes y tener encuentros culturales son deseos que no desaparecerán de la mente de los estudiantes, pues finalmente, reconocemos nuestras particularidades al reflejarnos en los otros.

\section{Referencias}

Altbach, P. \& De Wit, H. (7 de marzo de 2018). ¿Está en riesgo la internacionalización de la educación superior? Distancia por tiempos. Recuperado de https:// educacion.nexos.com. $\mathrm{mx} / \mathrm{p}=1047$

Altbach, P. \& De Wit, H. (Abril de 2020). Lo que pasaría con la educación superior tras la pandemia. Opinión, El Observatorio de la Universidad Colombiana. https://www.universidad.edu.co/lo-que-pasariacon-la-ed-sup-tras-la-pandemia-philip-altbachhans-de-wit-abril-20/

Altbach, P. G., Reisberg, L., \& Rumbley, L. E. (2009). Trends in global higher education: Tracking an academic revolution. https://doi.org/10.1163/9789004406155

AMPEI. (26 de mayo de 2020). Retos y oportunidades para la internacionalización de la educación superior (2/3) [Video]. YouTube. https://www.youtube.com/ watch?v=tO2wZPAOvUo\& $\mathrm{t}=344 \mathrm{~s}$

Appiah-Kubi, P., \& Annan, E. (2020). A Review of a Collaborative Online International Learning. International Journal of Engineering Pedagogy (iJEP), 10 (1), 109-124. https://doi. org/10.3991/ijep.v10i1.11678

Ascari, M. (17 de junio de 2016). How the young have forged European identity - from the Grand Tour to the Erasmus generation. The Conversation.

Barrón, M. (2020). La educación en línea. Transiciones y disrupciones. En H. Casanova (coord.). Educación y pandemia. Una visión académica. (pp. 66-74) México: Instituto de Investigaciones sobre la Universidad y la educación.

Beleen, J. (2011). La internacionalización en casa en una perspectiva global: un estudio crítico del Informe del 3er Estudio Global de la AIU RUSC. Universities and Knowledge Society Journal, 8(2), 85-100. http:// doi.org/10.7238/rusc.v8i2.1094

Calderon, A. (2018). Massification of higher education revisited. Melbourne: RMIT University. Recuperado de

https://www.academia.edu/36975860/
Massification_of_higher_education_revisited

Camacho, M. (2017). Internacionalización en la educación superior. Práctica y estudio en constante evolución vista desde un estudio de caso. México: ANUIES.

CEPAL y Unesco (2020). La educación en tiempos de COVID-19. Recuperado de https://repositorio.cepal. org/bitstream/handle/11362/45904/1/S2000510_ es.pdf

CIDRAP (2010). H1N1 \& Higher Ed: Lessons Learned Pandemic Influenza Tools, Tips, and Takeaways from the Big 10+2 Universities. Recuperado de https://www.cidrap.umn.edu/sites/default/files/ public/downloads/big102webfinal.pdf

De Wit, H. (2011). Globalización e internacionalización de la educación superior. RUSC. Universities and Knowledge Society Journal, 8 (2), 77-84. http://doi. org/10.7238/rusc.v8i2.1247

Donelly, M. y Gamsu, S. (2018). Home and away. Social, ethnic and spatial inequalities in student mobility. Reino Unido: University of Bath - The Sutton Trust.

Didou, S. (2013). Movilidades posgraduadas y científicas en México. En S. Didou (coord.), La formación internacional de los científicos en América Latina (pp. 87-163). México: ANUIES.

Didou, S. (2017). La internacionalización de la educación superior en América Latina: transitar de lo exógeno a lo endógeno. México: UDUAL.

Downing, K., Loock, P., \& Leung, H. (2 de marzo de 2018). The history and development of higher education ranking systems. QS WOW News. https:// qswownews.com/history-development-highereducation-ranking-systems/

Gacel-Ávila, J. (2017). Estrategias de internacionalización de la educación superior: implementación, evaluación y rankings. México: UNESCO - IESALC.

Gacel-Ávila, J. \& Rodríguez-Rodríguez, S. (2018). La internacionalización de la educación terciaria en América Latina y el Caribe: avances, tendencias y prospectiva. En J. Gacel-Ávila (coord.) La educación superior, internacionalización e integración regional de América Latina y el Caribe (pp. 57-88). Caracas: UNESCO-IESALC Y Córdoba: Universidad Nacional de Córdoba.

Gacel-Ávila, J. (2020). COVID-19: Riesgos y oportunidades para la internacionalización de la educación superior en México. ESAL Revista de Educación Superior en América Latina. 37-40. https://doi. org/10.14482/esal.8.378.721

Gómez, S. \& Colella, C. (2020). How COVID-19 has affected 
young universities. Bruselas: Young European Research Universities. https://www.yerun.eu/wpcontent/uploads/2020/07/YERUN-Covid-VFinalOnlineSpread.pdf

Góngora, E. (2018). Movilidad internacional, trayectorias y posicionamiento en tres sociólogos mexicanos. En R. Ramírez y J. Rodríguez (coords.). Internacionalización académica y científica: políticas, itinerarios, saberes e instrumentos (pp. 63-90). Ciudad de México: CINVESTAV.

Guth, S., Doscher, S. \& Haug, E. (mayo2020). Virtual Exchange and Global Learning Webinar organizado por Brazilian Virtual Exchange (BRaVE). Recuperado de http://erasmusplusriesal.org/es/noticias/webinarvirtual-exchange-and-global-learning

Hénard, F., Diamond, L., \& Roseveare, D. (2012). Approaches to Internationalisation and Their Implications for Strategic Management and Institutional Practice. OECD's Higher Education Programme. Recuperado de http://www.oecd.org/education/imhe/ Approaches\%20to\%20internationalisation\%20-\%20 final\%20-\%20web.pdf

Instituto Internacional de la UNESCO para la Educación Superior en América Latina y el Caribe (2019). La movilidad en la educación superior en América Latina y el Caribe: retos y oportunidades de un convenio renovado para el reconocimiento de estudios, títulos y diplomas. Recuperado de https:// unesdoc.unesco.org/ark:/48223/pf0000372629. locale $=$ es

Jaramillo, V. (2016). La cooperación Sur-Sur. En S. Didou (coord.), Reflexiones de expertos sobre la internacionalización de la educación superior (pp. 147-149). México: CINVESTAV - RIMAC.

Jung, N. (2016). Movilidad trasnacional posdoctoral y la vida después de la movilidad. ¿Me regreso, me quedo, o mejor no me hubiera ido? En R. Ramírez y M. Hamui (coords.) Perspectivas sobre la internacionalización en educación superior y ciencia (pp. 121 - 142). Ciudad de México: CINVESTAV.

Katz, R., May, L., Sanza, M., Johnston, L., \& Petinaux, B. (2012). H1N1 preventive health behaviors in a university setting. J Am Coll Health. 60 (1), 46-56. https://doi.org/10.1080/07448481.2011.570398

Knight, J. (2003). Updated definition of internationalization. International higher education, (33).

Knight, J. (s.f.). Capítulo 1 Internacionalización de la educación superior. Recuperado de https://www. ugto.mx/internacional/images/pdf/4a.pdf

Levinson, N. \& Davidson, K. (2015) “Linking Trajectories: On-line Learning and Intercultural Exchanges". International Journal for the Scholarship of Teaching and Learning, 9 (2), Article 3. https://doi. org/10.20429/ijsotl.2015.090203

Marmolejo, F. (2018). La educación superior en América Latina y el Caribe en el contexto global. En J. Gacel-Ávila (coord.) La educación superior, internacionalización e integración regional de América Latina y el Caribe (pp. 41-56). Caracas: UNESCO-IESALC y Córdoba: Universidad Nacional de Córdoba

OBIRET (2018). Resultados de la I Encuesta Regional sobre tendencias de la internacionalización en América Latina y el Caribe. Recuperado de http:// obiret-iesalc.udg.mx/es/noticias/resultados-dela-i-encuesta-regional-sobre-tendencias-de-lainternacionalizacion-en-america

OMS (2009). Medidas para los establecimientos escolares Gripe pandémica (H1N1) 2009. Nota informativa núm. 10. Recuperado de https://www.who. int/csr/disease/swineflu/notes/h1n1_school_ measures_20090911/es/

Oregioni, M., Taborga, A., \& Piñero, F. (2014). Proyección Integral de la Universidad Argentina hacia la Región Latinoamericana. Agenda, actores e instrumentos. III Jornadas de Extensión del mercosur. Tandil (Buenos Aires).

Pawlowska, E. (2011). El turismo académico: un análisis económico para el caso de Galicia. Compostela: Universidad de Santiago de Compostela (tesis de doctorado). Recuperado de https://minerva.usc.es/xmlui/ bitstream/handle/10347/3376/9788498877243_content.pdf;jsessionid=OD366F8D42E8695C5F08E55D6F9F09A4?sequence=1

Quinteiro, J. (19 de junio de 2020). ¿Cómo afectará el COVID -19 a la movilidad académica internacional? Blog UNESCO. Recuperado de https://www.iesalc. unesco.org/2020/06/19/como-afectara-el-covid-19-a-lamovilidad-academica-internacional/

Redden, E. (12 de mayo de 2009). Swine flu and student flows. Inside Higher Education. Recuperado de https:// www.insidehighered.com/news/2009/05/12/swine-fluand-student-flows

Remedi, E. \& Ramírez, R. (2016). La circulación del conocimiento en los procesos de formación, producción y comunicación científica. En S. Didou (coord.), Reflexiones de expertos sobre la internacionalización de 
la educación superior (pp. 87-91). México: CINVESTAV RIMAC.

Reynoso, C. (2010). La influenza A (H1N1) y las medidas adoptadas por las autoridades sanitarias. Desacatos, (32), 35-52.

Schleicher, A. (2020). The impact of COVID-19 on education: insights from Education at Glance 2020. Recuperado de https://www.oecd.org/education/the-impact-of-covid19-on-education-insights-education-at-a-glance-2020. pdf

Schwartzman, S. (2009). Nacionalismo versus Internacionalismo en las políticas de formación de recursos humanos de alto nivel. En S. Didou y Gerard, E. (coords.), Fuga de cerebros, movilidad académica y redes científicas. Perspectivas latinoamericanas (pp. 6373). México: IESALC - CINVESTAV - IRD.

Teichler, U. (2008). Student Mobility: Where Do We Come From, Where Are We, Where Are We Going to Inside the EHEA. En Presentation at the ACA Annual Conference.

Toro, D. (2020). Educación Superior en Latinoamérica en una economía post-COVID. ESAL Revista de Educación Superior en América Latina. 45-52. https://doi. org/10.14482/esal.8.378.8

Trucano, M. (16 de septiembre de 2014). Education and technology in an age of pandemics (revisited). World Bank Blogs. Recuperado de https://blogs.worldbank. org/edutech/education-technology-age-pandemicsrevisited

RIDU / Revista Digital de Investigación en Docencia Universitaria / e-ISSN: 2223-2516

(C) Los autores. Este artículo es publicado por la Revista Digital de Investigación en Docencia Universitaria del Área de Institutional Research and Effectiveness de la Dirección de Aseguramiento de la Calidad, Universidad Peruana de Ciencias Aplicadas. Este es un artículo de acceso abierto, distribuido bajo los términos de la LicenciaCreativeCommons Atribución-CompartirIgual 4.0 Internacional.( http://creativecommons.org/licenses/by-sa/4.0/), que permite el uso no comercial, distribución y reproducción en cualquier medio, siempre que la obra original sea debidamente citada. 
Gómez, A.

https://doi.org/ 10.19083/ridu.2020.1348

16

V. 14, n², jul-dic | PERÚ | 2020 
Gómez, A. 
Gómez, A.

https://doi.org/ 10.19083/ridu.2020.1348

18

V. 14, n², jul-dic | PERÚ | 2020 
Gómez, A.

https://doi.org/ 10.19083/ridu.2020.1348

19

V. 14, n², jul-dic | PERÚ | 2020 
Gómez, A.

https://doi.org/ 10.19083/ridu.2020.1348

20

V. 14, n², jul-dic | PERÚ | 2020 
Gómez, A. 
Gómez, A. 
Gómez, A. 
Gómez, A.

https://doi.org/ 10.19083/ridu.2020.1348

24

V. 14, n², jul-dic | PERÚ | 2020 
Gómez, A. 
Gómez, A.

https://doi.org/ 10.19083/ridu.2020.1348

26

V. 14, n², jul-dic | PERÚ | 2020 
Gómez, A.

https://doi.org/ 10.19083/ridu.2020.1348

27

V. 14, n², jul-dic | PERÚ | 2020 
Gómez, A.

https://doi.org/ 10.19083/ridu.2020.1348

28

V. 14, n², jul-dic | PERÚ | 2020 
Gómez, A.

https://doi.org/ 10.19083/ridu.2020.1348

29

V. 14, n², jul-dic | PERÚ | 2020 
Gómez, A.

https://doi.org/ 10.19083/ridu.2020.1348

30

V. 14, n², jul-dic | PERÚ | 2020 
Gómez, A.

https://doi.org/ 10.19083/ridu.2020.1348

31

V. 14, n², jul-dic | PERÚ | 2020 